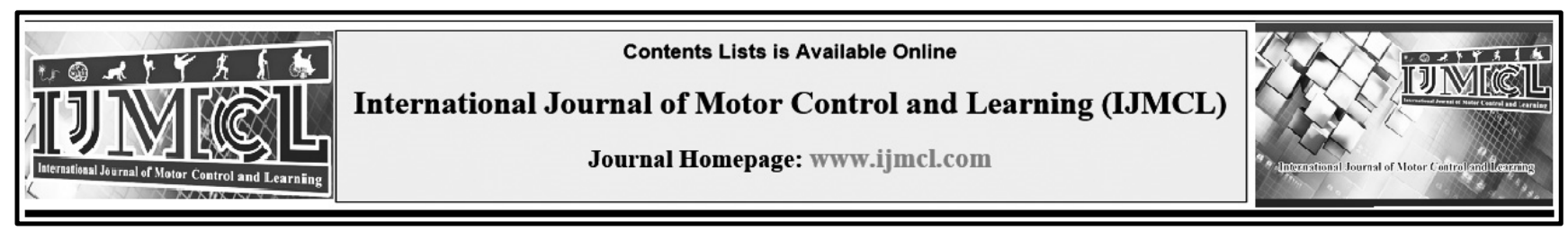

\title{
The Neurophysiological Effect of Attentional Focus as a Function of Expertise in Postural Task
}

\author{
Ali Pashabadi ${ }^{* a}$, Ali Ashraf Jamshidi ${ }^{\text {b }}$
}

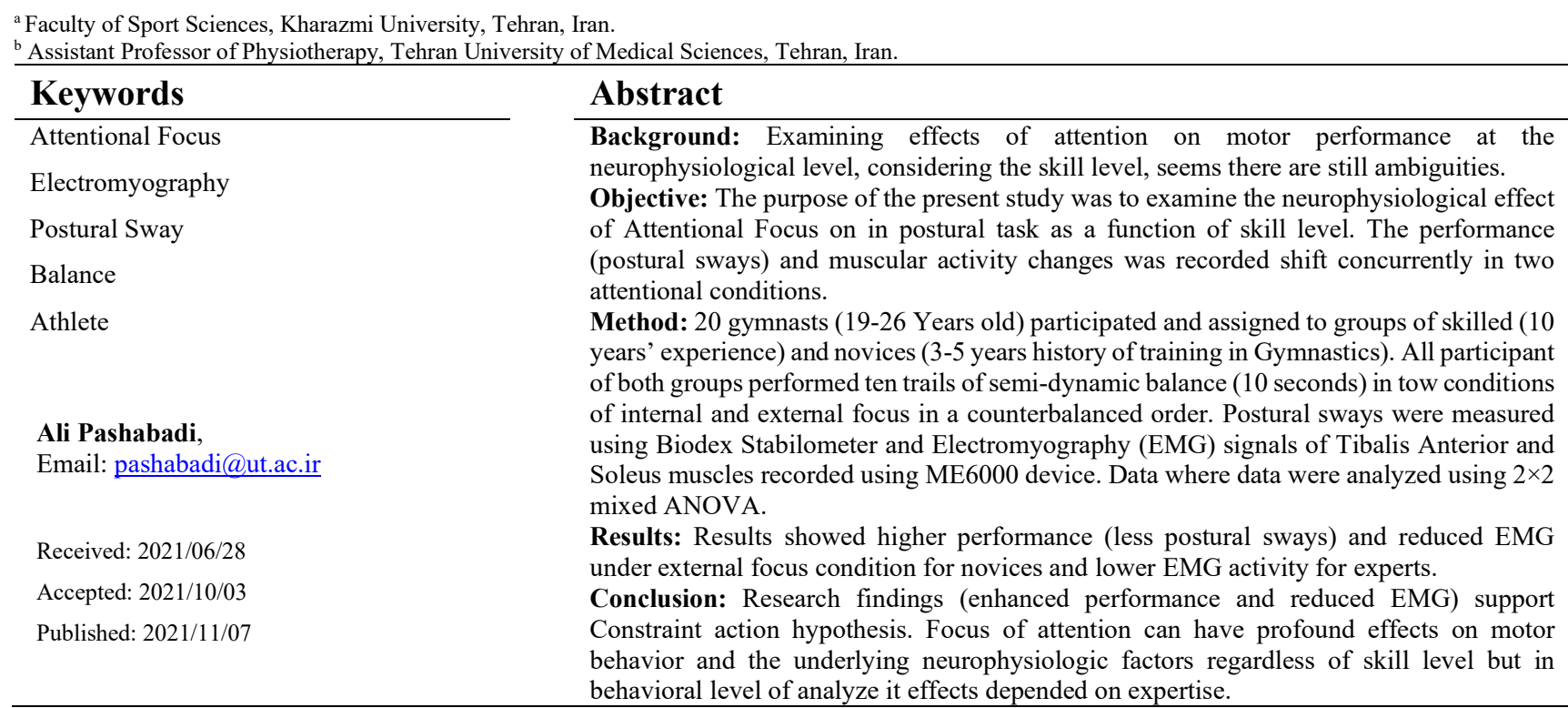

\section{Introduction}

Focus of attention while performing a motor skill is a determinant component which directed by instruction or feedback that performer receives (Wulf, 2013). The direction could be internal (focusing on one's own body movements), or external (focusing on the effects of the movement in the environment or its consequences) (Peh, Chow, \& Davids, 2011; Wulf, Shea, \& Lewthwaite, 2010). Numerous studies have revealed that focusing externally facilates the performance and learning of motor skills. Researchers have been shown that fluency, consistency, accuracy, and quality of the performance, and more important, it's result and outcome, are decidedly dependent on the performer focus of attention (Wulf, Shea, \& Park, 2001). Specifically, focusing on body or executive limbs, -adopting an external focus (In.F)-, while performing a motor skill has been found to be relatively ineffective. contrariwise, focusing on the movement's effects on the environment, has been verified to result in more effective performance and learning (Wulf, 2007, 2013). For example, external focus (Ex.F), has been found to enhance the performance in dart throw (Marchant, Clough, \& Crawshaw, 2007), baseball batting (Castaneda \& Gray, 2007), basketball free throw (Zachry, Wulf, Mercer, \& Bezodis, 2005); furthermore, there are some studies which showed balance performance 
(postural sways) improves (Wulf, Landers, Lewthwaite, \& To"1lner, 2009; Wulf, Mercer, \& McNevin, 2004; (Ellmers et al., 2016; N. McNevin, Weir, \& Quinn, 2013; N. H. McNevin \& Wulf, 2002; Olivier, Palluel, \& Nougier, 2008; Pashabadi, Farokhi, Jamshidi, \& Shahbazi, 2014; Richer, Saunders, Polskaia, \& Lajoie, 2017; Vuillerme \& Nafati, 2007; Wulf, Mercer, McNevin, \& Guadagnoli, 2004; Wulf, Töllner, \& Shea, 2007). Wulf and Prinz (2001) suggested Constraint Action Hypothesis to explain the benefits of Ex. F; given that when persons are instructed to adopt an In.F (or perhaps when there is no attentional instruction), attempt to control their movements consciously; therefore, and they constrain their movement system and unconsciously disturb the automatic control process of the action (Gabriele Wulf \& Wolfgang Prinz, 2001). Possible explanations as to why external focus produces superior outcomes include a reduced conscious control of movement inducing less "noise" in the motor system (Zachry et al., 2005) and efficient mechanisms underlying motor performance like electromyography (EMG). Earlier, Common Coding Hypothesis for the superiority of Ex.F (Gabriele Wulf \& Wolfgang Prinz, 2001) argued that we need to match coding system of efferent and afferent information during motor the performance. Particularly, he assumed that designing both perception and action is coded based on "remote events" of the body; that is distal events producing afferent and efferent codes and their common abstract representation (Wulf, 2007).

despite major body of evidence, there are some conflicts in the findings, and some studies, didn't found the advantage of Ex.F or in some cases the advantage of In.F, for instance, Beilock \& Carr (2002), Beilock \& Bertenthal (2004), PerkinsCeccato, Passamore and Lee (2003) and Uehara, Button and Davids (2008). These studies argue that the efficacy of attentional focus is dependent on the individual's experience. Most of the studies examining attentional focus and expertise level, have enclosed variables such as motor performance or learning, hence, -they have considered behavioral level of analysis- measured the "outcome" of the performance and paid less consideration to the Neurophysiologic level and measurement of performance "production" or the procedure of the movements. For example, EMG, provides a perspective how movement is controlled by neural system when people adopt various attentions and studies the neurophysiologic foundations of the movement. Vance, Wulf, Thomas, McNevin and Mercer (2004) concerning Neurophysiologic level and showed a reduced EMG activity when adopting Ex.F and this efficient muscular activity advantage (Vance, Wulf, Töllner, McNevin, \& Mercer, 2004) this findings also showed in other tasks; Marchant, Greig, Scott and Clough (2006) Isokinetic task, Zachry, Wulf and Mercer (2005) in the accuracy of basketball throw, Wulf, Dufek, Lozano and Pettigrew (2010) in high jump and Lohse, Sherwood and Healy (2010) dart throwing and currently (Ashraf, Aghdasi, \& Sayyah, 2017; Ay, Dolukan, \& Yıldiz, 2018). Despite this evidence, there are inconsistencies in the findings, when experts participate in attentional focus's experiments (Couvillion \& Fairbrother, 2018). 
Some studies confirmed the superiority of external focus, such as novices, and others have not observed a difference (Halperin, Chapman, Martin, \& Abbiss, 2017; Wulf \& Su, 2007), for experts, while others have found no difference in performance between conditions of focus, or, occasionally, superior performance under control conditions compared to both internal and external focus conditions (Porter \& Sims, 2013; Winkelman, Clark, \& Ryan, 2017; Wulf, 2008). Furthermore, research examining expert's attentional focus while performance has revealed that the adopting internal focus strategy is popular (Bernier, Codron, Thienot, \& Fournier, 2011; Guss-West \& Wulf, 2016). These findings together with the different findings from examination on skilled performers (Halperin et al., 2017; Porter \& Sims, 2013; Winkelman et al., 2017; Wulf, 2008; Wulf \& Su, 2007). Disclosed that attentional focus influences may not generalize to performer with high expertise level. Presumably, experts have developed attentional focus strategies that upkeep their top performance. Even though, some have contested (Guss-West \& Wulf, 2016), that expert's performance would be further enhanced through the systematic adoption of an Ex.F. Reviewing the literature showing a lack of advantage for top level performers suggests that such an approach is not yet merited. Balance task is required that the person in order to hold his/her postural control, needs continuous and sometime reflexive muscle activity. In addition, unlike Zachry et al. (2005) and Loshe et. al. (2010) tasks, muscles that are involved in balance task are grosser. While keeping the postural stability, how the EMG activity of involved muscles will be involved. The purpose of the current study was, therefore, to examine the effects of attentional focus as a function of expertise level, concurrently at the behavioral (performing balance task) and neurophysiologic (EMG activity) level of analysis. Two skilled and novice groups were included to typify potential ways in which responses to attentional instructions might vary for different skill levels.

\section{Method}

\section{Subjects}

The participants were 20 men gymnasts (19-26 years old), with right domain in lower extremity, normal posture and vision. They also had no musculoskeletal disorders, crucial injury, or fracture in lower limbs bones in the last two years. Based on experience they were assign to experimental groups of 10 skilled with at least 10 years' experience and 10 novices with between 3 to 5 years' history in gymnastic trainings.

\section{Apparatus and Task}

Postural sways were measured using The Biodex Balance System (Biodex Medical Systems, Inc., USA). The footboard of this stabilometer can be adjusted according to the level of difficulty. Task was keeping upright posture on unstable Biodex platform in semi-dynamic balance situation. The participants were instructed to stand on the footboard of unstable Biodex plate with their bare feet together and each of their hands holding their opposite shoulder and kept their eyes open. They had a couple of minutes to find their comfortable state before beginning trials. The verbal order 
"ready" and then "start" was given 3 seconds before the system began running, and at the end of the task, the Biodex plate changes from unstable to stable stance. Starting position was standing on stable platform, eyes open and keep looking forward. Participants performed ten trials of 20seconds with a 10 -seconds break between trails and 10 minutes break between blocks of 10 trials. The postural indices were calculated using the degree of oscillation of the platform, in which low values indicated that the individual had good stability. For each test conditions, the average of 20 trials was considered the subject score.

Surface EMG signals were acquired using EMG acquisition system (Me6000, Mega Electronics Ltd, Kuopio, Finland). Surface disposable $1.5 \mathrm{~cm}$ diameter Ag-AgCI electrodes containing silver chloride conductive jelly, was used with a centerto-center distance of $30 \mathrm{~mm}$.

\section{Procedure}

Participants came to Sports Sciences Research Center and after receiving basic clarifications by the researcher, got ready and warm up for performing the tasks. First, the location of the muscles on which electrodes were to be placed determined. Before the electrode's placement, the limb's skin was shaved and cleaned by alcohol pad and left to dry for $60 \mathrm{~s}$ to reduce the myoelelctrical impedance. To minimize the cross talk between the muscles, electrodes were placed carefully on the muscle belly and the protocols of electrode placement in this experiment followed the recommendations of SENIAM. The EMG signals were sampled at $1000 \mathrm{~Hz}$ and band pass filtered with cutoff frequencies of 10-500 Hz. Root Mean Square (RMS) of rectified and averaged signals was measured using Megawin 3.01 software. Raw data were normalized with a 1-second Maximum Voluntary Isometric Contraction (MVIC), and finally, these quantities were entering to statistical analysis.

Surface EMG activity signals of Tibialis Anterior (TA) and Soleus (SO) muscle recorded during balance trials. For the TA on the $1 / 3$ distance between the head of fibula and internal malleolus on the bulk of the muscle (Byrne, O'Keeffe, Donnelly, \& Lyons, 2007), and for the SO, $3 \mathrm{~cm}$ below the projection of the middle part of Gastrocnemius muscle in the medial part was electrodes were placed (Sacco, Gomes, Otuzi, Pripas, \& Onoderab, 2009; Rainoldi, Melchiorri, \& Caruso, 2004).

Both groups performed in counterbalanced order in tow attentional focus conditions (Vance et al., 2004). To control the effects of visual feedback, all the participants looked straight forward, before starting the measurement and in the breaks, they had time to look at the plate and monitor the position of their feet (McNevin et al, 2003). All the participants were told that the purpose is keeping their balance on the plate of Biodex stabilometer during the predetermined time is up, and they were not aware of the specific aims and details of the study.

\section{Data analysis}

After assumption met, data were analyzed using 2 (skill level: skilled vs novice) * 2 (attentional condition: Ex.F vs In.F) factorial ANOVA with 
repeated measure on last factor $(\mathrm{p}=0.05)$.

\section{Results}

The means and S.D for two groups of the skill levels measures during the tow conditions of attentional focus are presented in table. 1. The results for the stability index showed higher in skilled group, totally skilled had better performance score in balance task than novices.

Table 1. Mean \pm S.D. of Dependent Variable.

\begin{tabular}{llll}
\hline Dependent Variable & Skill level & Internal Focus & External Focus \\
\hline \multirow{2}{*}{ Postural Sway } & skilled & $1.93 \pm 0.47$ & $3.67 \pm 1.62$ \\
\cline { 2 - 4 } & novice & $1.85 \pm 0.69$ & $2.87 \pm 1.22$ \\
\hline \multirow{2}{*}{ EMG Tibialis Anterior } & skilled & $0.573 \pm 0.344$ & $0.401 \pm 0.27$ \\
\cline { 2 - 4 } & novice & $0.597 \pm 0.422$ & $0.538 \pm 0.39$ \\
\hline \multirow{2}{*}{ EMG Soleus } & skilled & $3.114 \pm 0.831$ & $2.740 \pm 0.99$ \\
\cline { 2 - 4 } & novice & $2.365 \pm 1.037$ & $2.803 \pm 0.84$ \\
\hline
\end{tabular}

A significant main effect attentional focus was found in the stability of both group's participants. More postural sways were observed in the Internal focus condition than in the External focus condition $(\mathrm{F}=16.22, \mathrm{P}<0.01, \eta 2 \mathrm{p}=0.26)$, indicating a higher degree of stability. Main effect of attentional focus on EMG activity was significant for both muscles including Tibialis Anterior $(\mathrm{F}=5.62, \mathrm{P}=0.03, \eta 2 \mathrm{p}=$ $0.40)$ and Soleus $(\mathrm{F}=18.76, \mathrm{P}=0.00, \eta 2 \mathrm{p}=0.51)$. For both group, the Tibialis Anterior and Soleus displayed significantly higher EMG activity ( $>0.05)$ during the Internal focus of attention as compared to Ex.F focus (Table 1).
Another portion of the study examined whether the effect of an attentional focus on postural sway varied as a function of skill level, and whether muscle cavity affected by this factor. Further analysis on factors interaction found a significant attention condition $\times$ expertise level interaction $(\mathrm{p}=.05)$ on postural sways $(\mathrm{F}=4.48, \mathrm{P}=0.04, \eta 2 \mathrm{p}=$ 0.20 ) but not significant for EMG activity of TA $(\mathrm{F}=1.35, \mathrm{P}=0.26, \eta 2 \mathrm{p}=0.07)$ and $\mathrm{SO}(\mathrm{F}=1.13$, $\mathrm{P}=0.30, \eta 2 \mathrm{p}=0.06$ ) muscle (Table.2.). Both groups increased their EMG activity across practice condition, with the internal focus tending to show somewhat higher activity.

Table 2. Results of mixed ANOVA with repeated measure test. Tests of Within-Subjects Effects

\begin{tabular}{|c|c|c|c|c|c|c|c|}
\hline & Source & $\begin{array}{l}\text { Type III Sum } \\
\text { of Squares }\end{array}$ & df & $\begin{array}{l}\text { Mean } \\
\text { Square }\end{array}$ & $\mathbf{F}$ & Sig. & $\eta 2 p$ \\
\hline \multirow{2}{*}{ Postural Sway } & Attentional Focus & 1.962 & 1 & 1.962 & 6.565 & 0.02 & 0.26 \\
\hline & Attentional Focus* Expertise & 1.31 & 1 & 1.31 & 4.484 & 0.04 & 0.20 \\
\hline \multirow{2}{*}{$\begin{array}{l}\text { EMG Tibialis } \\
\text { Anterior }\end{array}$} & Attentional Focus & 0.133 & 1 & 0.133 & 5.623 & 0.03 & 0.40 \\
\hline & Attentional Focus* Expertise & 0.032 & 1 & 0.032 & 1.354 & 0.26 & 0.07 \\
\hline \multirow{2}{*}{ EMG Soleus } & Attentional Focus & 0.621 & 1 & 0.621 & 18.764 & 0.000 & 0.51 \\
\hline & Attentional Focus* Expertise & 0.038 & 1 & 0.038 & 1.134 & 0.301 & 0.06 \\
\hline
\end{tabular}




\section{Discussion and Conclusion}

The purpose of this study was to examine effect of focus of attention on postural sways and EMG activity of leg muscles. Results showed that postural sways decreased in external focus, compared to internal and the findings denote those participants experienced less sways and a better semi-dynamic balance performance while focusing on external outcome of their performance. These findings along with most of the studies including Wulf, Töllner and Shea (2007), McNevin, Shea and Wulf (2003), Moghadam et al. (2008), Vuillerme et al. (2007). Higher stability in external focus can be explained commissioning Constraint Action Hypothesis. Any attempt to control the movement consciously, constrains the movement system and prevents the automatic processes that control the movement. Conversely, neglecting movement and focusing on its effects (EF) would allow the system to organize itself naturally (Wulf, 2007). Adopting an Ex.F allows unconscious, quick, and reflexive processing and appropriate results in the performance. On the other hand, in IF, participants control their movements consciously. Therefore, they intervene the natural processes of the movement system and, accordingly, constrain this system and unconsciously disturb their automatic control. In this way, automatic processes, which are able to control movement affectively are disturbed (Wulf, 2007). Perhaps this is more tangible in the balance and postural control of the athletes due to the development of their balance systems. This finding (improving the performance in external focus) supports Prniz's Common Coding hypothesis, in which, assumed that if actions are designed according to distal events or their conscious effects, they will be more affective (Gabriele Wulf \& Wolfang Prinz, 2001). So, participants' focus on the plate as compared to that of their legs makes less postural sways and improves the balance. As the results of interaction effect showed attentional focus in contrast with the novice group, had no significant effect in behavioral level of analysis for skilled performer. This could be results from a top performance that is near to the highest point of score so that there is no room to enhance performance anymore. This phenomenon is known in motor learning as the ceiling effect (Hirano, Sakurada, \& Furuya, 2020).

It is also pointed out in different studies that external focus in the participants with less skill is facilitating and allows these people to have a performance as high as those in upper levels (Wulf, Dufek, Lozano, \& Pettigrew, 2010). The superiority of external focus in the novice was contradictory with some findings, of Beilock et al. (2002 \& 2004), Perkins-Ceccato et al, (2003), and Uehara et al, (2008); perhaps this is related to the level of participant's skill and stages of learning. In the aforementioned studies, participants were in the early stages of learning; they need declarative knowledge and attention to the duration of the performance. They also benefit from directing attention to movement. But the participants in the current study had a 3-5-year experience, and probably could handle challenging situations. They have reached higher levels of learning and focusing on the processed procedures while performing could be harmful to them. 
The results of comparing EMG in TA and SO muscles in attentional conditions, showed the reduced EMG activity in EF. These findings support Vance et al. (2004), Zachry et al. (2005), Wulf et al. (2010), and Lohse et al. (2010). Former studies have considered the decrease of muscle activities as economic and better efficacy in movement (Vance et al., 2004; Zachry et al., 2005), as well as the improvement of the neuromuscular efficacy in generating movement (Lohse, Sherwood, \& Healy, 2010). These results represent that to focusing the outcome of task provides better efficiency in muscle activity of same task. This means that Ex.F creates movement that consumes less energy than In.F (Wulf et al, 2010; Marchant et al, 2006). In present study, also, the slight EMG activity of TA and SO while controlling posture with Ex.F can corroborate such idea. The findings supported Constraint Action Hypothesis (Vance et al., 2004; Wulf, 2007) too. According to this hypothesis, focusing on the action itself (In.F) constrains the aforementioned factors and leads to producing noise in the activity of the muscles. This increase in EMG is evidence of the amplification in muscular stiffness and the reduction in efficacy while internal focus (Lohse et al., 2010).

The Ex.F instruction can accompny by optimized pattern of involved muscles in the determined task (between muscles coordination) and increased within muscles coordination (the pattern of the motor unit recruitment in a muscle) reduces the stiffness of the muscle (Lohse et al, 2010; Wulf et al, 2010). Better performance with hiegher energy efficiency in completing a specific outxome defined as the task goal coulad be effectiveness of attentional instructions, therefore, improvement in the efficacy of motor unit's recruitment is beneficial for most of the motor tasks. Effective recruitment of muscle fibers in a muscle (within muscle coordination), and increasing the coordination among muscle groups are advantageous in performing the task. They cause useful issues; such as generating appropriate force (maximum, if necessary) at appropriate time and in right direction. Furthermore, activities requiring endurance should benefit from less and optimized neuromuscular activity for the specific outputs. This saves energy or maintained the specific level of the activity for longer durations (Zachry et al., 2005).

Increased efficacy and efficiency of the movement is typically observed in higher skill levels and advanced learning stages. Researches, however, suggest that giving the external instruction of attentional focus makes the participant to show a higher performance level in a specific stage of learning (Lohse et al, 2010). The current findings support this claim. It is believed that focusing on the desirable outputs of movement creates a neuromuscular pattern in the performer, which is similar to the patterns in the performances by those in higher skill levels. As we know early in the weight training, increase in strength occurs as the function of the training maybe it's not explainable by just hypertrophy (Hodson-Tole \& Wakeling, 2009). It is also reported that the reduced EMG with lifting the same weight after some sessions is due to the increase in the efficacy of muscle in the movement production. It is commonly believed that neuromuscular adaptation 
is the fundamental reason for this phenomenon (Lohse et al, 2010). This decrease in EMG was obviously observed in this study. Of course, it has been suggested that several other factors, such as task requirements, are effective in recruitment pattern. Generally, when considering the recent work of Hudson-tol and Vakling (2009), the compatibility between influential factors in the motor unit's recruitment is not thoroughly recognized. In any event, it is necessary to add the participant's focus to the list of the factors that affect the neuromuscular activity patterns (Lohse et al, 2010; Wulf et al, 2010).

The simultaneous decrease in postural sways (improved performance) and reduced EMG activity (improved efficiency), were similar to the findings of Zachry et al. (2005), Lohse et al, (2010) (enhanced accuracy and reduced EMG), and Wulf et al. (2010) (enhanced height of jumping and reduced EMG). Increase of noise in motor system (higher EMG) because of In.F hinder the fine control of the movement and reduces the reliability of its outputs (Zachry et al., 2005). The effective pattern of the movement as a result Ex.F causes the increase of accuracy and eventually improves the consequence of movement (Bernier et al., 2011; Lohse et al., 2010; Porter \& Sims, 2013; Winkelman et al., 2017). They point that in EF; neuromuscular activity is reduced and motor unit recruitment occurs more distinctly. According to literature adopting Ex.F can facilitate the posture compensatory adjustments during the task to keep the effects of the movement (keeping the stability of posture). On the other hand, focusing on the movement itself can have opposite effects, for example the increase of the muscular stiffness at the expense of the movement consequence (Calatayud et al., 2018; Lohse et al., 2010). In this study, we observed the increase in postural sways with reduced EMG activities of the leg muscles. As this investigation and previous ones concerning the attentional focus revealed, even slight differences in the structure of the task (in this case, small modification in saying the instructions) can have a profound effect on the movement structure and its foundational neurophysiologic factors. Thus, trainers, instructors, therapists and practitioners need to be aware that the shift in focus of attention can affect in both behavioral and neurophysiologic levels. So, they must develop the strategies of keeping the focus of the participant externally. Therefore, we recommend the trainers when giving the instructions and feedbacks directing the focus of attention to consider the skill level of the participant. Our findings are only applicable to the novice or those with 3-5 years of experience. For other levels, we should approach the issue with caution and according to the results of other studies.

\section{References}

1. Ashraf, R., Aghdasi, M. T., \& Sayyah, M. (2017). The effect of attentional focus strategies on children performance and their EMG activities in maximum a force production task. Turkish Journal of Kinesiology, 3(2), 26-30.

2. Ay, A. N., Dolukan, Y. B., \& Yıldız, M. Z. (2018). The effect of attentional focus conditions on performer's EMG activity. Academic Perspective Procedia, 1(1), 240-247.

3. Bernier, M., Codron, R., Thienot, E., \& Fournier, J. F. (2011). The attentional focus of expert golfers in training and competition: a naturalistic investigation. Journal of Applied Sport Psychology, 23(3), 326-341. 
4. Byrne, C. A., O'Keeffe, D. T., Donnelly, A. E., \& Lyons, G. M. (2007). Effect of walking speed changes on tibialis anterior EMG during healthy gait for FES envelope design in drop foot correction. Journal of Electromyography and Kinesiology, 17, 605-616.

5. Calatayud, J., Vinstrup, J., Jakobsen, M. D., Sundstrup, E., Colado, J. C., \& Andersen, L. L. (2018). Influence of different attentional focus on EMG amplitude and contraction duration during the bench press at different speeds. Journal of sports sciences, 36(10), 1162-1166.

6. Castaneda, B., \& Gray, R. (2007). Effects of focus of attention on baseball batting performance in players of differing skill levels. Journal of Sport and Exercise Psychology, 29(1), 60-77.

7. Couvillion, K. F., \& Fairbrother, J. T. (2018). Expert and novice performers respond differently to attentional focus cues for speed jump roping. Frontiers in Psychology, 9, 2370.

8. Ellmers, T. J., Machado, G., Wong, T. W.-L., Zhu, F., Williams, A. M., \& Young, W. R. (2016). A validation of neural co-activation as a measure of attentional focus in a postural task. Gait \& Posture, 50, 229-231.

9. Guss-West, C., \& Wulf, G. (2016). Attentional focus in classical ballet: a survey of professional dancers. Journal of Dance Medicine \& Science, 20(1), 23-29.

10. Halperin, I., Chapman, D. W., Martin, D. T., \& Abbiss, C. (2017). The effects of attentional focus instructions on punching velocity and impact forces among trained combat athletes. Journal of sports sciences, 35(5), 500-507.

11. Hirano, M., Sakurada, M., \& Furuya, S. (2020). Overcoming the ceiling effects of experts' motor expertise through active haptic training. Science advances, 6(47), eabd2558.

12. Hodson-Tole, E. F., \& Wakeling, J. M. (2009). Motor unit recruitment for dynamic tasks: current understanding and future directions. J Comp Physiol B, 79, 79:57-66.

13. Lohse, K. R., Sherwood, D. E., \& Healy, A. F. (2010). How changing the focus of attention affects performance, kinematics, and electromyography in dart throwing. Human movement science, 29(4), 542-555.

14. Marchant, D. C., Clough, P. J., \& Crawshaw, M. (2007). The effects of attentional focusing strategies on novice dart throwing performance and their task experiences. International Journal of Sport and Exercise Psychology, 5(3), 291303.

15. McNevin, N., Weir, P., \& Quinn, T. (2013). Effects of attentional focus and age on suprapostural task performance and postural control. Research quarterly for exercise and sport, 84(1), 96-103.

16. McNevin, N. H., \& Wulf, G. (2002). Attentional focus on supra-postural tasks affects postural control. Human movement science, 21(2), 187-202.

17. Olivier, I., Palluel, E., \& Nougier, V. (2008). Effects of attentional focus on postural sway in children and adults. Experimental Brain Research, 185(2), 341-345.

18. Pashabadi, A., Farokhi, A., Jamshidi, A., \& Shahbazi, M. (2014). Effect of attentional focus on postural sways and muscular activity in athletes. Modern Rehabilitation, 8(1).

19. Peh, S. Y.-C., Chow, J. Y., \& Davids, K. (2011). Focus of attention and its impact on movement behaviour. Journal of Science and Medicine in Sport, 14(1), 70-78.

20. Porter, J. M., \& Sims, B. (2013). Altering focus of attention influences elite athletes sprinting performance. International Journal of Coaching Science, 7(2).

21. Richer, N., Saunders, D., Polskaia, N., \& Lajoie, Y. (2017). The effects of attentional focus and cognitive tasks on postural sway may be the result of automaticity. Gait \& Posture, $54,45-49$.

22. Vance, J., Wulf, G., Töllner, T., McNevin, N., \& Mercer, J. (2004). EMG activity as a function of the performer's focus of attention. Journal of motor behavior, 36(4), 450-459.

23. Vuillerme, N., \& Nafati, G. (2007). How attentional focus on body sway affects postural control during quiet standing. Psychological Research, 71(2), 192-200.

24. Winkelman, N. C., Clark, K. P., \& Ryan, L. J. (2017). Experience level influences the effect of attentional focus on sprint performance. Human movement science, 52, 84-95.

25. Wulf, G. (2007). Attentional focus and motor learning: A review of 10 years of research. EJournal "Bewegung und Training, 1, 1-64.

26. Wulf, G. (2008). Attentional focus effects in balance acrobats. Research quarterly for exercise and sport, 79(3), 319-325.

27. Wulf, G. (2013). Attentional focus and motor learning: a review of 15 years. International Review of Sport and Exercise Psychology, 6(1), 77-104.

28. Wulf, G., Dufek, Janet S, Lozano, L., \& Pettigrew, C. (2010). Increased jump height and reduced EMG activity with an external focus. Human Movement Science, 29, 440-448.

29. Wulf, G., Mercer, J., McNevin, N., \& Guadagnoli, M. A. (2004). Reciprocal influences of attentional focus on postural and 
suprapostural task performance. Journal of motor behavior, 36(2), 189-199.

30. Wulf, G., \& Prinz, W. (2001). Directing attention to movement effects enhances learning: A review. Psychonomic Bulletin \& Review, 8 (4), 648-660.

31. Wulf, G., \& Prinz, W. (2001). Directing attention to movement effects enhances learning: A review. Psychonomic bulletin \& review, 8(4), 648-660.

32. Wulf, G., Shea, C., \& Lewthwaite, R. (2010). Motor skill learning and performance: a review of influential factors. Medical education, 44(1), 75-84.

33. Wulf, G., Shea, C., \& Park, J.-H. (2001). Attention and motor performance: preferences for and advantages of an external focus. Research quarterly for exercise and sport, 72(4), 335-344.

34. Wulf, G., \& Su, J. (2007). An external focus of attention enhances golf shot accuracy in beginners and experts. Research quarterly for exercise and sport, 78(4), 384-389.

35. Wulf, G., Töllner, T., \& Shea, C. H. (2007). Attentional focus effects as a function of task difficulty. Research quarterly for exercise and sport, 78(3), 257-264.

36. Zachry, T., Wulf, G., Mercer, J., \& Bezodis, N. (2005). Increased movement accuracy and reduced EMG activity as the result of adopting an external focus of attention. Brain research bulletin, 67(4), 304-309. 